PU-RCG/99/19

\title{
Are pre-big-bang models falsifiable by gravitational wave experiments?
}

\author{
Carlo Ungarelli ${ }^{1}$ and Alberto Vecchio \\ Max Planck Institut für Gravitationsphysik, Albert Einstein Institut \\ Am Mühlenberg 1, D-14476 Golm, Germany
}

\begin{abstract}
One of the most interesting predictions of string-inspired cosmological models is the presence of a stochastic background of relic gravitational waves in the frequency band accessible to Earth-based detectors. Here we consider a "minimal" class of string cosmology models and explore whether they are falsifiable by gravitational wave observations. In particular, we show that, the detectability of the signal depends crucially on the actual values of the model parameters. This feature will enable laser interferometers - starting from the second generation of detectors - to place stringent constraints on the theory for a fairly large range of the free parameters of the model.
\end{abstract}

\section{MOTIVATIONS}

One of the direct phenomenological predictions of inflationary cosmological models is the generation of a stochastic background of gravitational waves (GW's). For "slow-roll" inflationary models this prediction is hardly testable: since the spectrum is almost flat over the huge frequency band $\sim 10^{-16} \mathrm{~Hz}-1 \mathrm{GHz}[1]$, in the window $10 \mathrm{~Hz}-1 \mathrm{kHz}$, where ground-based GW experiments operate, the maximum value of the energy spectrum $\Omega_{\mathrm{gw}}(f) \equiv \rho_{c}^{-1} d \rho_{\mathrm{gw}}(f) / d \ln (f)$ compatible with the COBE measurements of the cosmic micro-wave background (CMB) temperature fluctuations at large scales $-h_{0}^{2} \Omega_{\mathrm{gw}} \leq 10^{-14}[2]$ - is well below the sensitivity expected for the third generation of detectors, $h_{0}^{2} \Omega_{\mathrm{gw}} \gtrsim 10^{-11}$.

"Pre-big-bang" (PBB) models [3] represent an alternative to the standard "slow-roll" inflationary scenario. In a minimal PBB model, one assumes that the initial state is the perturbative vacuum of super-string theory, the flat 10-dimensions Minkowski space-time; the Universe goes first through an inflationary phase where the curvature and the string coupling increase, eventually reaches a "stringy epoch" where the curvature scale is of order one in units of the string length, and finally evolves into a typical decelerated radiation $/ \mathrm{matter}$ dominated era. The inflationary PBB phase has a precise consequence on the structure of $\Omega_{\mathrm{gw}}(f)$, which affects the detectability of the stochastic background by laser interferometers, whose "science-runs" are expected to begin at the end of 2001: in the low frequency range, the spectrum is characterized by a steep power law, $\Omega_{\mathrm{gw}} \propto f^{3}$ [4]; indeed the COBE bound is easily evaded, and the spectrum can peak at frequencies of interest for GW experiments, while satisfying the existing experimental bounds $[5,6]$.

For the rather general class of minimal PBB models, $\Omega_{\mathrm{gw}}(f)$ depends on two free parameters: (i) the redshift $z_{s} \equiv f_{1} / f_{s}$ of the high-curvature phase, which fixes the "knee" frequency $f_{s}$ between the low and high frequency regime $\left(f_{1} \sim 10^{10} \mathrm{~Hz}\right.$ is the cut-off frequency of the spectrum, whose exact value is irrelevant for the issues discussed here); (ii) the value $g_{s}$ of the string coupling at the onset of the high-curvature phase, which determines the high-frequency slope of the spectrum. In Fig. 1 we show $\Omega_{\mathrm{gw}}(f)$ for two choices of the free parameters; varying $g_{s}$ and $z_{s}$, the maximum value of the spectrum compatible with the constraints due to pulsar timing data and to the abundance of light elements at the nucleosynthesis epoch is $h_{0}^{2} \Omega_{\mathrm{gw}} \sim 10^{-7}[6]$. $\Omega_{\mathrm{gw}}(f)$ strongly depends on $z_{s}$ and $g_{s}$ : they affect both the frequency behaviour and the peak value of the

1) Present address: School of Computer Science and Mathematics, University of Portsmouth, Mercantile House, Hampshire Terrace, Portsmouth P01 2EG, UK 


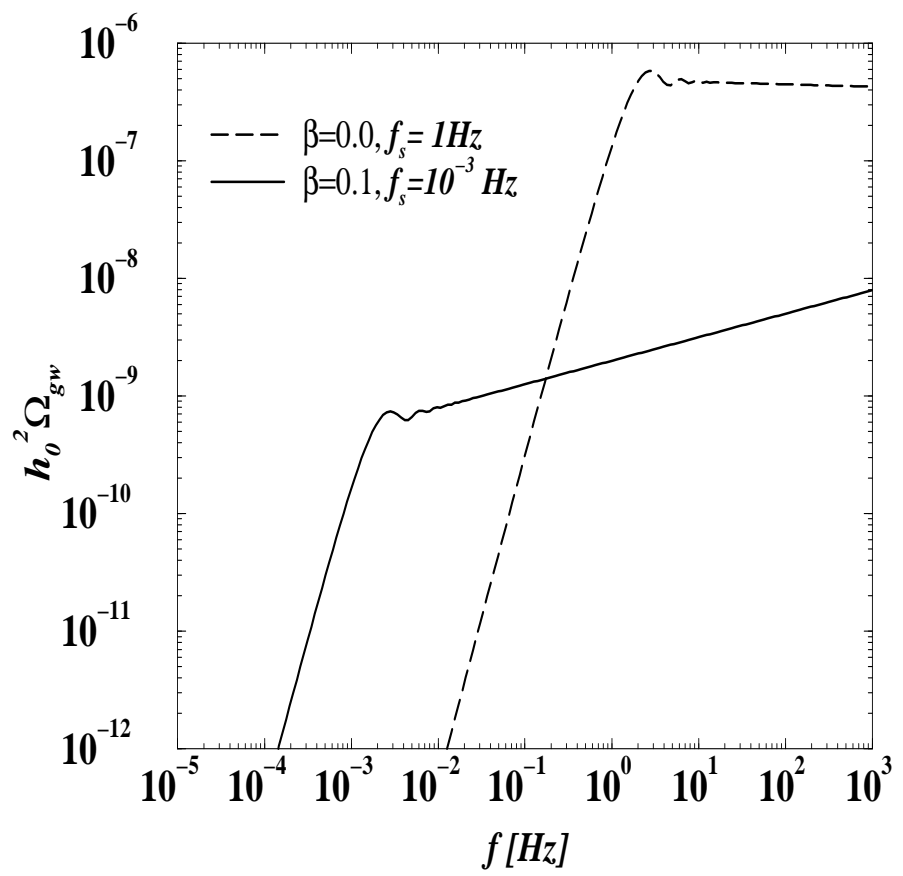

FIGURE 1. The energy spectrum $h_{0}^{2} \Omega(f)$ of relic gravitons according to the prediction of minimal pre-big-bang models as a function of the frequency (in $\mathrm{Hz}$ ) for two different choices of the model parameters; here the parameter $\beta$ is defined as $\beta=-\frac{\log \left(g_{s} / g_{1}\right)}{\log z_{s}}$.

spectrum. Whether GW experiments will be able or not to detect a signal predicted by PBB models does depend on the actual value of the "true" parameters. Indeed, GW experiments represent one of the very few avenues where the cosmological models can be verified, and could open a new era for studies of the very-early Universe and the structure of fundamental fields at high energies $[7,8]$. The emphasis of this contribution is not on finding the best theoretical scenario which would guarantee a detection. The goal of our analysis is to address to which extent GW observations can test PBB models, and more in detail to identify the the region of the free-parameter space that experiments can probe. A similar analysis, in the context of string cosmology models, was carried out in [9].

\section{RESULTS}

In order to address whether a background characterized by a given energy spectrum $\Omega_{\mathrm{gw}}\left(f ; z_{s}, g_{s}\right)$ is indeed detectable or not, one needs to evaluate the signal-to-noise ratio (SNR) that can be obtained by cross-correlating the output of two detectors as a function of the model parameters, in our case $z_{s}$ and $g_{s}$ (see also [9]). The data analysis issues related to the detection of a stochastic background have been thoroughly discussed in [10], where the reader can find full details. Here we recall only the expression of the signal-to-noise ratio:

$$
\mathrm{SNR} \approx \frac{3 H_{0}^{2}}{10 \pi^{2}} T_{\mathrm{obs}}^{1 / 2}\left[\int_{-\infty}^{\infty} d f \frac{\gamma^{2}(|f|) \Omega_{\mathrm{gw}}^{2}\left(|f| ; z_{s}, g_{s}\right)}{f^{6} S_{1}(|f|) S_{2}(|f|)}\right]^{1 / 2} .
$$

In Eq. (1) $T_{\text {obs }}$ is the observation time - which is usually assumed to be a few months long $-H_{0}=100 h_{0} \mathrm{~km}$ $\mathrm{sec}^{-1} \mathrm{Mpc}^{-1}$ is the Hubble constant, $S_{i=1,2}(f)$ is the noise spectral density of the $i$-th detector, and $\gamma(f)$ is the overlap reduction function. The spectrum $\Omega_{\mathrm{gw}}\left(f ; g_{s}, f_{s}\right)$ for minimal PBB models is given in [6].

Fig. 2 summarizes the results: it shows the contour plots of SNR for $T_{\mathrm{obs}}=10^{7} \mathrm{sec}$ which can be achieved by cross-correlating the two 4-km LIGO interferometers located in Hanford and Livingston. The contours are drawn in the relevant 2-dimensional parameter space: in order to highlight the physical content, our parameter choice corresponds to $\log _{10}\left(z_{s}\right)$ and $\log _{10}\left(g_{s} / g_{1}\right)$; here $g_{1}^{2} / 4 \pi=\alpha_{G U T}$, where $\alpha_{G U T}$ is the gauge coupling at the 


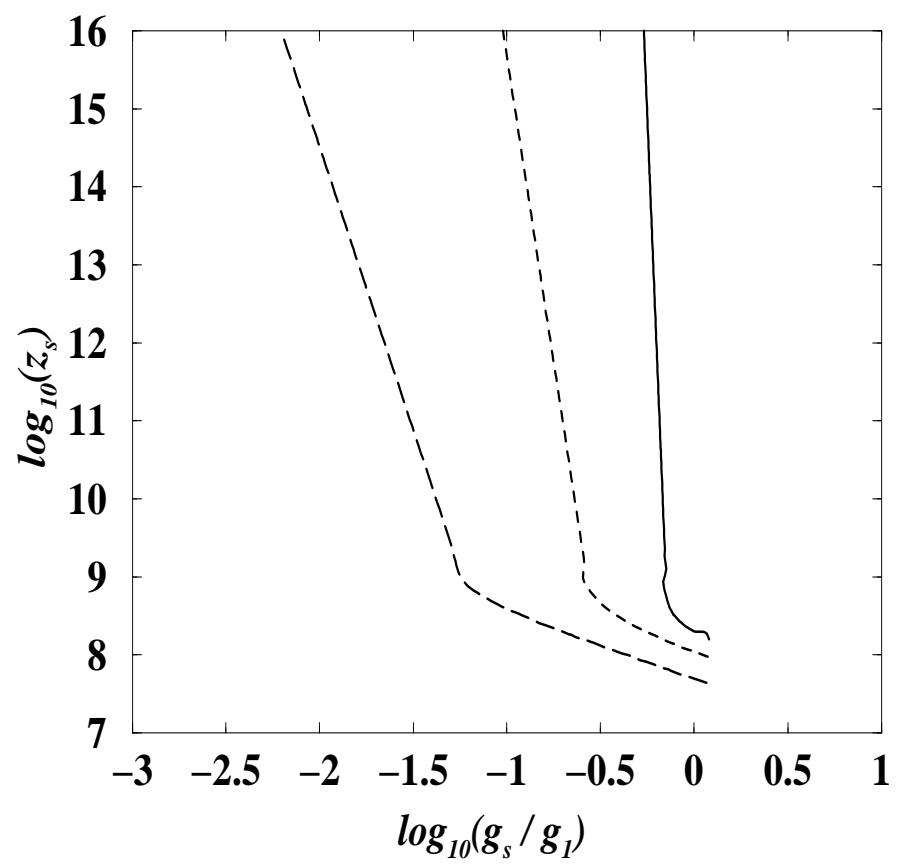

FIGURE 2. The signal-to-noise ratio (SNR) produced by the GW stochastic background predicted by minimal pre-big-bang models. The figure shows the contour plots of the SNR in the plane of the free-parameters of the model, $f_{s}$ and $g_{s}$, obtained by cross-correlating the two LIGO interferometers. The curves corresponds to: SNR $=0.1$ for the initial LIGO configuration (solid line); $\mathrm{SNR}=5$ for the enhanced LIGO configuration (short-dashed line); SNR $=10$ for the advanced LIGO configuration (long-dashed line). We remind that the physical range over which the parameters are defined is: $0<g_{s} / g_{1}<1$ and $1<z_{s}<10^{16}$.

unification scale $\left(\alpha_{G U T} \sim 1 / 20\right)$. The two free-parameters of the models are defined over the following range: $0<g_{s} / g_{1}<1$ and $1<z_{s}<10^{16}$. In our analysis we have considered the estimated noise spectral density for the initial, enhanced and advanced LIGO configuration (the so-called LIGO I, II, and III, respectively).

For initial LIGO, the minimum value of a detectable stochastic background is $h_{0}^{2} \Omega_{\mathrm{gw}}^{\min } \sim 5 \times 10^{-6}$, cfr. also [10], and therefore the experiments can not provide any direct hint about PBB models: the maximum value of SNR, for fined-tuned parameters of the model, is just below one. However, the enhanced LIGO configuration will allow us to explore a relatively large parameter region: for SNR > 5, one can probe models where $g_{s}$ differs by one order of magnitude with respect to its present value $g_{1}$ and about eight orders of magnitude in $f_{s}$; the $\mathrm{SNR}$ reaches a maximum $\sim 100$ when $g_{s} \simeq g_{1}$. The advanced LIGO configuration - which however requires major technological developments - will enlarge the $g_{s}$-range that one can test by more than one order of magnitude; for selected parameter values the experiment could reach SNR $\approx 1000$. Notice that the experiments are very sensitive to $g_{s}$, and achieve the maximum SNR for $g_{s} \sim g_{1}$.

One might wonder whether other interferometers and/or resonant detectors could provide relevant information for the problem at hand. Unfortunately the location, orientation and sensitivity of VIRGO, GEO600 and TAMA are such that any search involving one of these three instruments will not allow to detect a stochastic background of PBB gravitons; just as reference, a VIRGO-GEO600 cross-correlation experiment will reach $h_{0}^{2} \Omega_{\mathrm{gw}}^{\min } \sim 8 \times 10^{-6}$ for an integration time of 4 -months. Neither resonant antenna are suitable: two "bars" operating at the quantum limit with exactly the same resonance frequency would reach a maximum sensitivity $h_{0}^{2} \Omega_{\mathrm{gw}}^{\mathrm{min}} \sim 5 \times 10^{-6}$ (see also [11]), which is a factor $\sim 10$ worse than the one required for the most optimistic theoretical prediction; experiments involving hollow spheres [12], which are currently under study, could reach $h_{0}^{2} \Omega_{\mathrm{gw}}^{\min } \sim 10^{-7}$ for detectors operating at the quantum limit and co-located; this would produce a SNR $\sim 1$ for a PBB model whose parameters are such that the peak of $\Omega_{\mathrm{gw}}(f)$ is right in the middle of the sphere frequency band. 


\section{CONCLUSIONS}

Minimal PBB models predict a GW stochastic background which could be detectable by cross-correlating the two LIGO instruments - or, more in general, two interferometers at a distance $<3000 \mathrm{~km}$ and quasioptimally oriented - with a sensitivity which is intermediate between the first and second stage. In the time frame 2004-2005, when the detectors are expected to operate in the so-called enhanced configuration, we will be able to place experimental constraints on PBB models for a fairly large portion of the free-parameter space. A more detailed analysis regarding the issues discussed here is currently in preparation.

\section{REFERENCES}

1. B. Allen, Phys. Rev D 37, 2078 (1988). A. Liddle and M. Turner, Phys. Rev. D 50, 758 (1994); M. Turner, Phys. Rev. D 55, 435 (1997).

2. L. Krauss and M. White, Phys. Rev. Lett. 69, 869 (1992).

3. G. Veneziano, Phys. Lett. B265, 287 (1991); M. Gasperini and G Veneziano, Astropart. Phys. 1, 317 (1993), Mod. Phys. Lett. A8, 3701 (1993).

4. R. Brustein, M. Gasperini, M. Giovannini and G. Veneziano, Phys. Lett. B 361, 45 (1995).

5. R. Brustein, M. Gasperini and G. Veneziano, Phys. Rev. D 55, 3882 (1997).

6. A. Buonanno, M. Maggiore, C. Ungarelli, Phys. Rev. D 55, 3330 (1997).

7. T. Creighton, preprint gr-qc/9907045.

8. M. Maggiore, to appear in Physics Reports, preprint gr-qc/9909001.

9. B. Allen and R. Brustein, Phys. Rev. D 55, 3260 (1997).

10. B. Allen and J.D. Romano, Phys. Rev. D 59, 102001 (1999).

11. S. Vitale, M. Cerdonio, E. Coccia and A. Ortolan Phys. Rev. D 55, 1741 (1997).

12. E. Coccia, V. Fafone, G. Frossati, J. A. Lobo and J. A. Ortega Phys.Rev. D 57, 2051 (1998). 